\title{
La grande vitesse ferroviaire et la transformation spatiale urbaine : le cas de Saragosse (Espagne)
}

High-speed rail and urban spatial transformation: the case of Zaragoza (Spain)

Die Hochgeschwindigkeitsstrecke und die räumliche Veränderung: Der Fall

Zaragoza (Spanien)

Carmen Bellet Sanfeliu

\section{(2) OpenEdition}

Electronic version

URL: http://journals.openedition.org/rge/5566

ISSN: $2108-6478$

\section{Publisher}

Association des géographes de l'Est

Printed version

Date of publication: 1 October 2015

ISSN: 0035-3213

\section{Electronic reference}

Carmen Bellet Sanfeliu, « La grande vitesse ferroviaire et la transformation spatiale urbaine : le cas de Saragosse (Espagne) », Revue Géographique de l'Est [Online], vol.55 / n³-4 | 2015, Online since 13 October 2015, connection on 08 September 2020. URL : http://journals.openedition.org/rge/5566

This text was automatically generated on 8 September 2020

Tous droits réservés 


\section{La grande vitesse ferroviaire et la transformation spatiale urbaine : le cas de Saragosse (Espagne)}

High-speed rail and urban spatial transformation: the case of Zaragoza (Spain) Die Hochgeschwindigkeitsstrecke und die räumliche Veränderung: Der Fall Zaragoza (Spanien)

\section{Carmen Bellet Sanfeliu}

\section{Introduction ${ }^{1}$}

1 La planification et construction des réseaux ferroviaires à grande vitesse en Espagne, comme cela l'a été en France et d'autres pays européens, est un puissant argument pour développer deux types d'intervention dans les villes :

2 - L'évolution du système ferroviaire qui permet d'améliorer l'intégration de ses espaces dans la trame urbaine en palliant le rôle traditionnel de barrière que les installations ferroviaires ont depuis toujours joué (Bertolini y Spit, 1998; Bruinsma, et al. 2008; Van den Berg and Pol, 1998).

3 - Les réalisations urbanistiques plus ou moins ambitieuses autour de la gare à grande vitesse (neuve ou remodelée) en tant qu'argument pour générer de nouvelles centralités urbaines ou pour renforcer/redynamiser celles existant déjà (Bertolini et Spit, 1998; Debrezion, Willigiers, 2008; Peters, Novy, 2012)

4 L'arrivée d'une nouvelle ligne à grande vitesse est en général accompagnée d'une reconsidération/nouvelle mise en oeuvre complète de l'équipement ferroviaire (réseau et installations) sur le territoire et dans la ville (Groupe TEN, 1993). La remise en œuvre non seulement tente d'améliorer la fonctionnalité et l'efficacité du système de transport mais aussi la rentabilité économique des espaces ferroviaires plus ou moins centraux. En fait, les plus-values urbanistiques qui sont le résultat d'opérations de transformation ferroviaire, ont été une des sources de financement les plus 
importantes des projets d'implantation du nouveau train. Ainsi, à échelle locale, de nombreuses administrations ont accueilli le train avec de grands espoirs car cela représentait d'une part la connexion à une infrastructure de transport de grande capacité, mais aussi l'occasion de procéder à une importante transformation urbaine. En fin, une opportunité stratégique pour la ville. Le projet du nouveau chemin de fer est alors, dans certaines occasions, devenu un instrument urbanistique de premier ordre arrivant à transformer la structure de l'ensemble urbain. (Bellet, Gutierrez, 2011).

Pour tenter d'intégrer l'infrastructure dans le milieu urbain il existe des solutions douces (traitement des bords, augmentation de la perméabilité, construction de passages à différents niveaux, adaptation aux conditions topographiques du lieu, entre autres) et des solutions dures (construction en souterrain de la traversée urbaine ou la construction d'une variante du tracé). En Espagne et avec l'implantation du nouveau réseau, on a opté pour les solutions maximalistes et dures en étudiant la déviation du tracé ou en choisissant de rendre souterraine la traversée ferroviaire urbaine. Ces solutions de limitation de l'encombrement urbain reviennent très cher. Les plus-values immobilières générées par la libération du sol d'usage ferroviaire doivent financer une bonne partie du projet. La solution qui passe par la construction d'une déviation du tracé, éloignant la gare des centres urbains (excepté dans le cas de déviation extérieure) est, d'après différents auteurs, difficilement compatible avec la fonction de moyen de transport de voyageurs dont l'efficacité est fondée sur la centralité de ses gares (Troin, 1995).

6 Malgré tout, les forts investissements qu'occasionnent les positions centrales et les minutes qui peuvent être perdues dans les trajets entre de grandes gares terminales font que, dans les lieux moins peuplés, la localisation de la gare qui doit accueillir les services de la grande vitesse soit périphérique. Dans ce contexte, les critères d'efficacité de la grande vitesse ferroviaire pèsent sur la décision de l'emplacement. Cela est le cas d'un bon nombre de gares françaises (Vendôme, Mâcon-Loche, Le Creusot, Haute Picardie, Arbois, Marne-la Vallée, Satolas, Rhône-Alpes Sud en Valence, etc.) (Menerault, 2007; Facchinetti-Mannone, 2005) et seulement quelques cas en Espagne (Guadalajara-Yebes, Segovia-Guiomar y Camp de Tarragona) (Ureña, 2006; Ribalaygua, 2005)

7 Le volume des investissements est souvent élevé et l'on espère en financer une bonne partie grâce aux plus-values générées par les processus de transformation et/ou libération de sol occupé auparavant par les infrastructures ferroviaires. Les société, et les projets qu'elles entreprennent, doivent être autofinancés c'est-à-dire que les rentes obtenues de la vente du sol libéré doivent couvrir une bonne partie du coût des opérations ce qui n'est pas seulement un risque pour la viabilité du projet mais aussi une importante limitation pour le réaménagement urbanistique qui, dans la plupart des cas, promet d'être stratégique. La forte récession du marché immobilier commencée en 2008, à laquelle s'ajoute aujourd'hui l'état généralisé de la dette publique, complique pour ces sociétés le développement des projets initialement prévus arrivant même à compromettre leur exécution.

Dans ce contexte, l'objectif de cet article est d'analyser la relation entre l'arrivée d'une ligne à grande vitesse et la transformation de Saragosse. Nous faisons l'hypothèse que le train peut devenir un instrument de dynamisation s'il existe une cohérence entre le projet d'implantation du nouveau système ferroviaire et le modèle urbain local (défini dans le plan urbanistique et, au cas où il existerait, le plan stratégique de la ville). Le 
rôle et les stratégies déployées à échelle locale par les différents agents du processus sont aussi analysés. L'analyse des documents de planification stratégique et territoriale, des entretiens faits avec des techniciens locaux et une documentation venant de la presse locale constituent les principales sources d'information.

\section{La transformation urbanistique et l'arrivée du TGV dans les villes espagnoles}

9 L'arrivée du TGV en Espagne a commencé en 1992 avec l'inauguration du premier tronçon Madrid-Séville dans le couloir sud qui arrive désormais jusqu'à Malaga. Le processus de grande vitesse ferroviaire s'est accéléré de façon intense dans les années 2000 avec de nouvelles lignes reliant différentes villes. À la suite d'une politique extensive et ambitieuse d'encouragement des infrastructures, l'Espagne disposait fin 2013 de $3.100 \mathrm{~km}$ de voies de chemin de fer à grande vitesse et de 31 gares en fonctionnement (Bel et Albalate, 2012).

10 La longue expérience européenne montre que l'arrivée de la grande vitesse ferroviaire a été accompagnée d'importantes opérations de transformation urbaine (Van Den Berg et Pol, 1998; Bruinsma et al., 2008). Pourtant, il semble que l'Espagne soit le pays européen où l'arrivée du TGV ait été accompagnée des changements les plus intenses dans la forme et la structure physique des villes (Bellet, Alonso et Gutiérrez, 2012). Les raisons en sont nombreuses, comme par exemple:

11 - L'introduction du train à grande vitesse dans la ville a représenté une grande opportunité pour faire face aux problèmes historiques d'intégration du chemin de fer dans la ville. En Espagne, la relation entre le chemin de fer et la ville a presque toujours été conflictuelle. L'arrivée du nouveau train a donc servi à remodeler l'ensemble du système ferroviaire, entraînant des transformations urbanistiques importantes. Dans de nombreux cas, il s'agit des travaux de rénovation ou de transformation urbaine les plus importants dans l'histoire de chaque ville. Il en est ainsi parce que lors de l'implantation du nouveau train, l'Espagne semble avoir fait un choix qui, dans la majorité des cas, comporte des solutions d'intégration maximalistes (et souvent plus coûteuses) : l'enfouissement des voies ferrées ou la construction de nouveaux tracés extérieurs.

12 - Dans certaines villes, les travaux d'implantation du TGV ont été utilisés pour résoudre d'autres problèmes urbanistiques hérités ou non résolus, même si certains n'ont pas ou très peu de relations avec le nouveau train, comme par exemple: résoudre des besoins d'équipement des quartiers, trouver des solutions pour la mobilité métropolitaine, restructurer les centralités urbaines, etc.

13 - L'arrivée du TGV a coïncidé avec une époque d'expansion du cycle immobilier et avec des prévisions de croissance économique très favorables dans l'ensemble du pays. Ces prévisions étaient nourries des projets de TGV, autour desquels ont surgi une multitude de projets immobiliers.

14 Dans certains cas, l'arrivée du train est devenue un prétexte pour concevoir de grands projets, comme c'est le cas avec la ville nouvelle de Valdeluz. Il s'agit d'une nouvelle opération immobilière planifiée autour de la nouvelle gare périphérique, où une nouvelle ligne et une gare TGV ont été construites à $8 \mathrm{~km}$ du centre-ville de Guadalajara et à 20 minutes de Madrid par le nouveau train. Une nouvelle ville 
«intégrale» d'après les promoteurs, pour 30.000 personnes, avec un total de 9.500 logements planifiés. Le train a été mis en service en 2003, mais en 2010, seul un terrain de golf avait été construit et 1.500 logements avaient été livrés à leurs propriétaires. La ville compte actuellement environ 3.000 habitants (Prada, 2010). Une opération immobilière clairement spéculative. Le cas de Valdeluz est devenu l'un des symboles les plus représentatifs des effets territoriaux de l'expansion du secteur de la construction en Espagne pendant les années 2000 et de l'explosion ultérieure de la bulle immobilière en 2008 .

Figure 1 : Lignes espagnoles à grande vitesse ferroviaire et situation des gares, janvier 2014

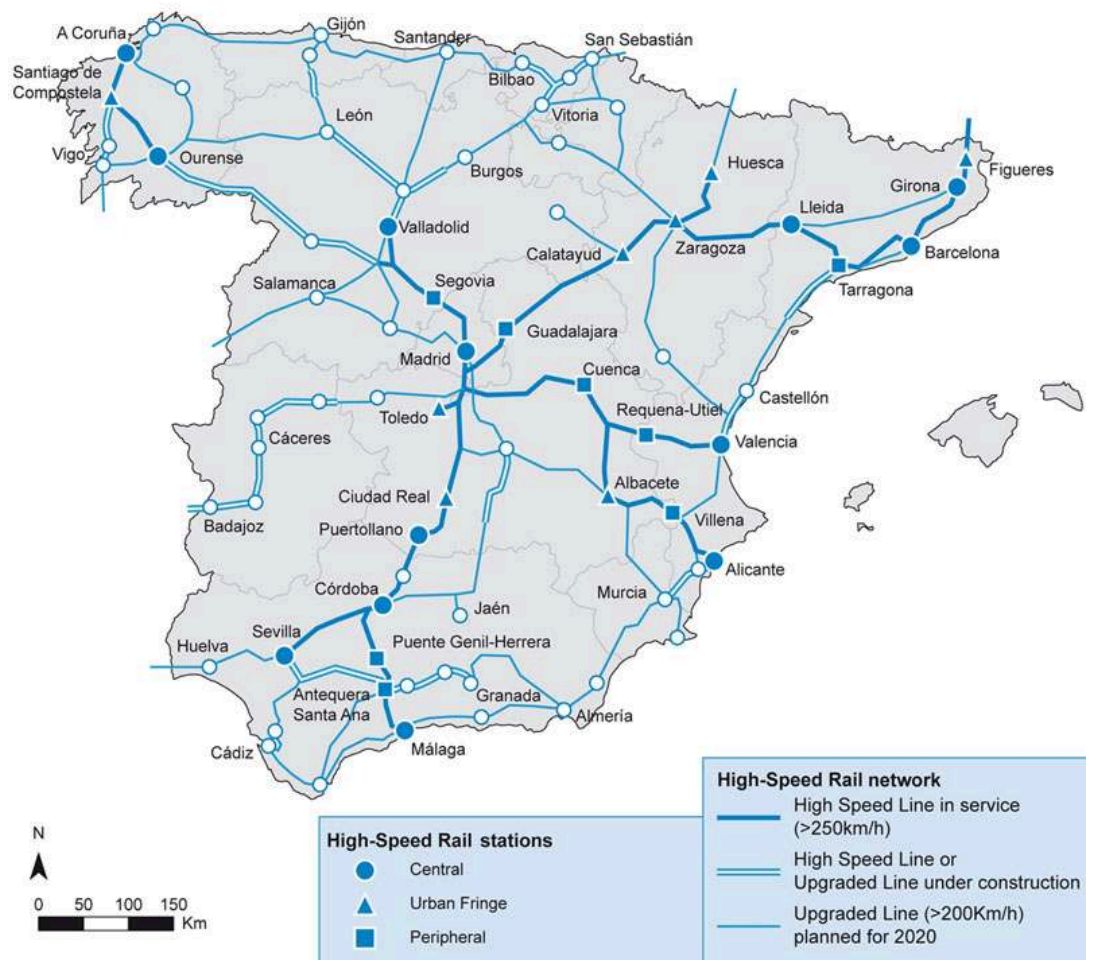

Source: Élaboration personnelle

15 Compte tenu de ce contexte, il est facile de comprendre pourquoi l'arrivée du TGV a entraîné, dans les villes et sur l'ensemble du territoire espagnol, de grands projets immobiliers (Bellet, Alonso, Gutiérrez, 2012).

Il existe d'autres cas intéressants, pour lesquels l'implantation du nouveau train, avec une gare centrale ou périphérique, est associée à une restructuration importante du système ferroviaire, avec par exemple la création de nouveaux tracés tangentiels, le démantèlement d'éléments ferroviaires centraux, etc. Ceci implique une vision totalement différente de l'ensemble de la structure urbaine. Parmi les villes qui ont élaboré des projets de transformation urbaine en Espagne, le plus important et le plus intéressant est celui de la ville de Saragosse. En voici les principales raisons (Alonso, Bellet, 2009; Ureña, Menerault, Garmendia, 2009) :

- Tout d'abord, parce que la restructuration ferroviaire (les modifications à réaliser sur le système ferroviaire existant) a été très importante et a entraîné des changements structurels favorisant l'apparition d'une nouvelle structure urbaine. 
18 - Ensuite, parce que l'arrivée du TGV a été perçue dans la ville comme un instrument d'accompagnement d'un nouveau projet urbain. Un nouveau projet urbain ambitieux qui cherche à générer des changements profonds pour dynamiser le territoire et améliorer son image sur le plan national et international.

19 - Dès le début, les agents locaux (l'association d'architectes, les ingénieurs, la chambre de commerce et d'industrie locale, les entrepreneurs) ont participé à la phase de planification de l'infrastructure, en choisissant les modalités d'implantation du TGV. Par conséquent, le modèle d'implantation ferroviaire était très cohérent avec le modèle de ville. Le projet urbain sur lequel les agents locaux ont travaillé a guidé l'implantation de l'infrastructure, ce qui a permis l'apparition d'une ville pratiquement nouvelle.

20 En définitive, le TGV est devenu un instrument clé pour le projet urbain de la Saragosse du XXIe siècle et c'est ainsi qu'il a été mis en évidence dans tous les documents de planification physique et stratégique de la ville.

\section{Saragosse: le TGV et les transformations urbaines}

21 Saragosse, une ville de près de 700.000 habitants (682.004 selon le recensement municipal au premier janvier 2013), est l'une des communes les plus étendues du pays avec $973,78 \mathrm{~km} 2$; elle est située dans un endroit géostratégique, dans le nord-est de la péninsule et dans la vallée de l'Èbre, à $300 \mathrm{~km}$ de Madrid et de Barcelone, mais aussi à environ $300 \mathrm{~km}$ de Bilbao et de Toulouse.

22 La ville a un caractère clairement administratif et tertiaire; il s'agit en fait de la capitale de la région (Communauté autonome d'Aragon). Les activités industrielles, notamment celles liées aux véhicules à moteur et aux industries auxiliaires (Opel est l'une des entreprises les plus importantes de la région) ont également joué un rôle important dans la dynamique économique de la zone métropolitaine. À ces activités se sont ajoutées récemment les activités logistiques qui profitent des systèmes de production flexibles et de la position géostratégique de la ville dans les principaux couloirs du nord-est péninsulaire (Arnal et al., 2009).

23 Saragosse cherche également depuis quelques années à se défaire de son image de ville grise, sans attraits, traditionnelle et assez conservatrice.

\section{A. Les instruments du changement urbain à Saragosse}

Pour cela, la ville a préparé un ensemble de plans et de projets qui ont abouti avec plus ou moins de succès à la conception d'un projet urbain, un modèle sur lequel travailler. Un plan stratégique a été conçu au début des années 1990, l'un des plus cohérents et des mieux gérés d'Espagne, approuvé en 1998 et qui a été révisé récemment. La rédaction du plan stratégique (Ebropolis) coïncide avec la rédaction du nouveau plan général d'aménagement urbain (approuvé en 2001), et aussi avec la rédaction d'un ensemble de plans et de projets qui semblent tous partager la volonté de construire une nouvelle stratégie pour la métropole, un nouveau projet urbain pour Saragosse. Parmi ceux-ci, l'un des projets les plus importants est celui abordant l'implantation du TGV. Un peu plus tard, la célébration d'un événement international, l'Exposition 
internationale de 2008 sur le thème de l'eau, a signifié des progrès importants dans le positionnement international de la ville et dans les fonctions de celle-ci (Serrano, 2009). mission était de concevoir et de promouvoir le plan stratégique pour l'ensemble de la zone métropolitaine. Les membres fondateurs étaient très variés et comprenaient des administrations locales et régionales, la chambre de commerce locale, des associations commerciales, l'université locale, les principaux syndicats de la ville, des associations de quartiers et plusieurs organismes bancaires. Le premier plan, approuvé en juillet 1998, définissait le modèle de la future ville et a obtenu un large consensus parmi les parties impliquées. Cependant, la plus grande réussite de l'association et du plan a peut-être été la création d'un rêve collectif pour un changement urbain, avec une grande cohérence interne et sans pratiquement aucune critique contre la proposition. Ou du moins cela a été le cas jusqu'à l'arrivée de la crise économique en 2008. Le brouillon du plan général, approuvé en 2001, et le projet de lancement du TGV, qui était déjà débattu à l'époque, devait s'adapter parfaitement aux lignes directrices déjà évoquées par le plan stratégique.

33 - Les organismes professionnels techniques, en particulier le Colegio Oficial de Arquitectos de Aragon (l'Ordre officiel des architectes d'Aragon), ont également joué 
un rôle décisif dans la définition du projet $\mathrm{du} \mathrm{TGV}$, comme nous le verrons ci-après. Lorsque les techniciens du ministère des Travaux publics ont présenté leurs idées initiales pour l'intégration locale du TGV, ils ont imaginé une perspective locale qui était très différente de celle qui avait été débattue si vivement et approuvée au niveau local. Cette nouvelle vision était fondée sur les propositions des architectes locaux, et qui ont été finalement adoptées.

34 - Zaragoza Alta Velocidad (Saragosse grande vitesse): Un accord de partenariat a été signé en 2002 entre trois administrations: le ministère des Travaux publics, la Diputación General de Aragón (le gouvernement régional) et la mairie de Saragosse pour assurer la gestion, le financement et la mise en œuvre des travaux de transformation ferroviaire et urbanistique Les objectifs de l'accord se résument de la façon suivante: gérer le développement urbanistique du sol qui n'aurait plus un usage ferroviaire et exécuter les travaux des nouvelles infrastructures ferroviaires ainsi que ceux nécessaires à son bon fonctionnement. Pour la mise en œuvre de ces objectifs, une société à capitaux publics a été créée: Zaragoza Alta Velocidad (Saragosse grande vitesse). La Société est chargée de: l'engagement et de l'exécution des travaux, de faire des propositions urbanistiques et d'organiser la vente des terrains constructibles pour le financement des opérations.

\section{Le projet ferroviaire à Saragosse comme instrument urbanistique}

Les premières propositions d'implantation du TGV prévoyaient d'installer le train à grande vitesse dans la gare centrale classique de Portillo, que l'on voulait transformer en une grande gare intermodale (la super-gare largement évoquée dans la presse). Cependant, des approches différentes avaient été envisagées, comme celle proposée par l'Association des architectes d'Aragon. Celle-ci indiquait que le choix de Portillo n'était pas le plus adéquat, car d'une part il générait une concentration excessive dans une zone déjà saturée et, d'autre part, il empêchait de faire de l'arrivée du nouveau train un instrument d'aménagement urbain de premier ordre.

Après de rudes négociations, la proposition qui a été retenue est le projet alternatif prévoyant des changements considérables dans le système ferroviaire local à partir de trois grandes interventions :

1. La fermeture de la gare traditionnelle centrale de Portillo.

2. La création d'une grande gare intermodale à l'ouest de la ville à la place de l'ancienne gare de marchandises dans la zone de Delicias.

3. La construction d'un by-pass ferroviaire dans le sud de la ville pour y bâtir la nouvelle gare de marchandises. Ces travaux sont actuellement les plus avancés. 
Figure 2 : L'implantation du TGV à Saragosse.

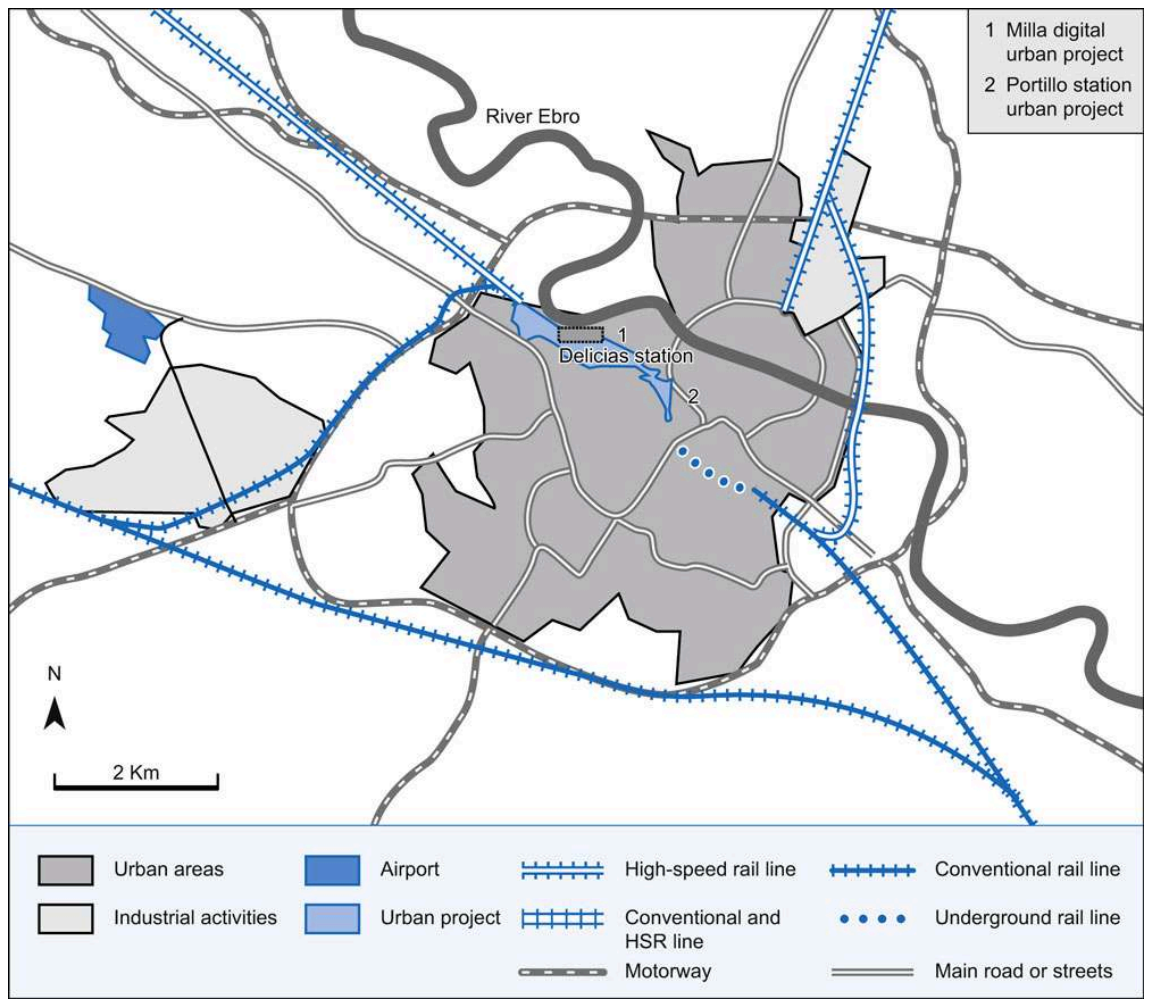

Source : Élaboration personnelle

\section{A. Le by-pass ferroviaire extérieur et la plateforme logistique PLAZA}

Sur la Figure 2, le by-pass extérieur situé au sud de la ville, sur lequel la nouvelle gare de marchandises est en cours de construction, près de l'aéroport, du parc des expositions et de la Plateforme Logistique de Saragosse (Plaza). La plateforme, comme nous l'avons indiqué ci-dessus, est devenue l'une des plus grandes aires logistiques du sud de l'Europe, avec près de 1.300 hectares d'où opèrent des sociétés telles que Inditex (Zara), Dhl Express, le siège de stockage de données de la Barclays Bank, des sociétés de construction et de carrelages comme Porcelanosa, etc. L'intermodalité de la plateforme logistique apporte une grande valeur ajoutée grâce au déplacement de la gare de marchandises vers cette zone.

Ce tracé ferroviaire extérieur est également utilisé par les trains qui circulent sur la ligne Madrid-Barcelone sans s'arrêter à Saragosse. Le gouvernement aragonais a lancé un appel d'offres pour la construction d'une deuxième gare de TGV sur le by-pass, près de la gare de marchandises, pour que les trains circulant en ligne directe entre Madrid et Barcelone puissent également faire un arrêt à Saragosse. Cependant, la gare n'a pas encore été construite, et l'anneau ferroviaire au sud n'a pas été achevé. Ce chantier a dû être interrompu à cause de la survenue de la crise.

\section{B. Définition d'une nouvelle centralité pour Portillo}

Au centre, comme nous l'avons vu, la gare classique de Portillo a été fermée et déplacée vers la zone auparavant occupée par l'ancienne gare de marchandises de Delicias. La 
désaffectation ferroviaire de Portillo a permis de restructurer la zone et d'en faire une nouvelle aire urbaine centrale, de 9,3 hectares, en contact direct avec le centre-ville, dans un contexte de centralité classique.

Le plan de développement du site, approuvé en 2005, envisage de construire des équipements culturels de premier ordre: Caixaforum (centre d'expositions et d'événements culturels), un auditorium et une médiathèque. En outre, le projet inclut une gare de banlieue (déjà en fonctionnement), un centre de santé et un immeuble de bureaux et de logements. Le projet s'appuie essentiellement sur un nouvel aménagement constitué en grande partie d'espaces libres et de zones vertes. (45.000 $\mathrm{m} 2)$.

Figure 3 : La transformation urbanistique à Portillo et à Delicias

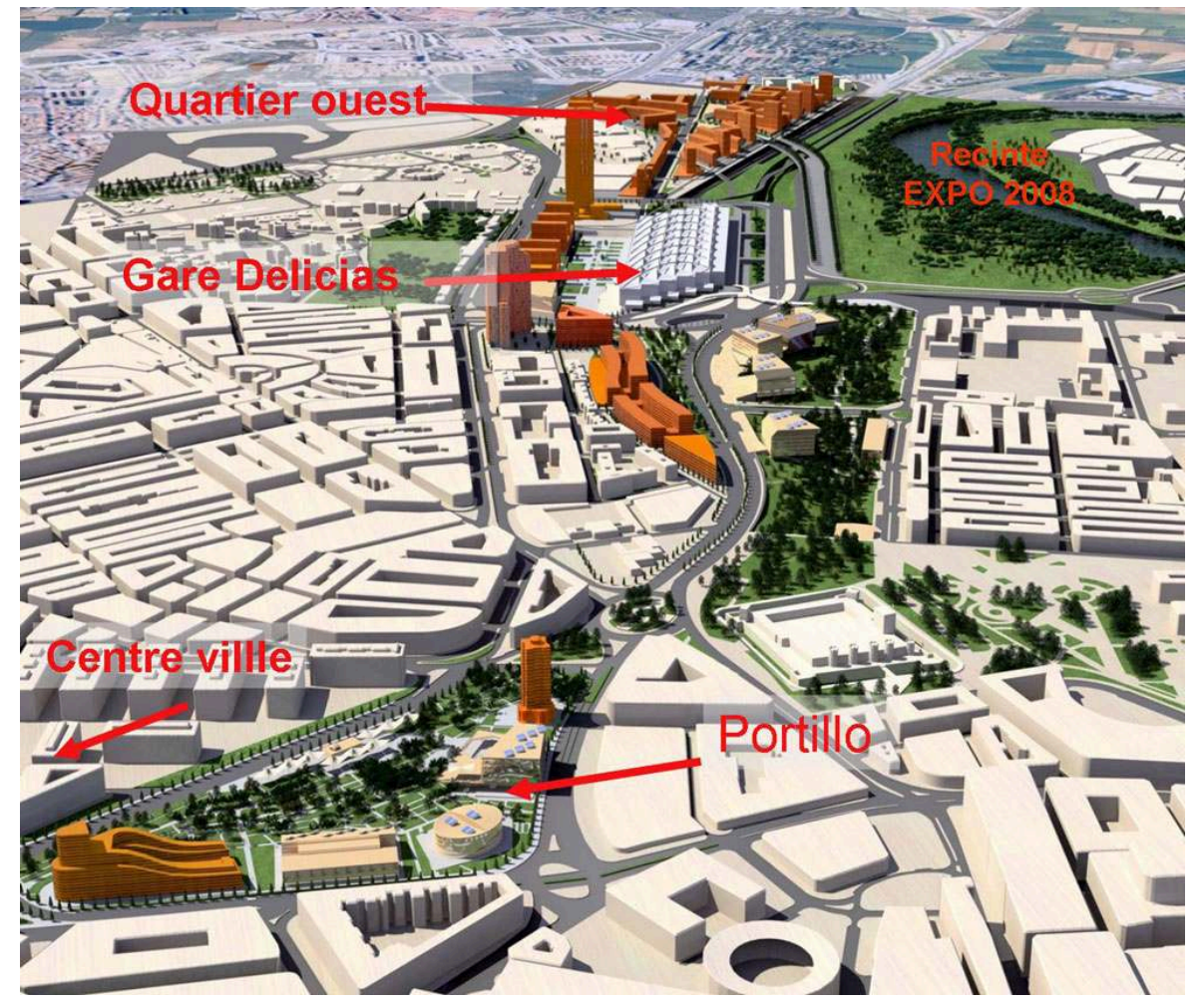

Source : Zaragoza Alta Velocidad

\section{Les travaux dans le secteur de Delicias-Almozara}

41 La deuxième intervention centrale consistait en la construction d'une nouvelle gare dans la zone de Delicias. Cette opération permettrait à la ville de gagner environ 95,8 hectares à l'ouest de la ville, où le sol était, en grande partie, ferroviaire. Le plan urbanistique consistait essentiellement à ouvrir cette partie de la ville au fleuve, à articuler les quartiers de l'ouest métropolitain (Delicias et Almozara, séparés auparavant par des barrières infrastructurelles) et de prolonger la centralité du centreville jusqu'à la gare.

42 Sur la Figure 4, la gare de Delicias, qui était déjà terminée en 2003 lorsque les premiers TGV sont arrivés dans la ville. Cette gare a été conçue comme un aéroport avec une zone d'arrivées et de départs et des accès aux trains très contrôlés. Il s'agit d'un grand 
bâtiment qui inclut la gare intermodale avec des services de train conventionnel, les nouveaux trains de banlieue et les TGV, en plus d'une gare routière. La gare comprend aussi un hôtel, des commerces et des restaurants.

Figure 4 : La gare intermodale Saragosse-Delicias

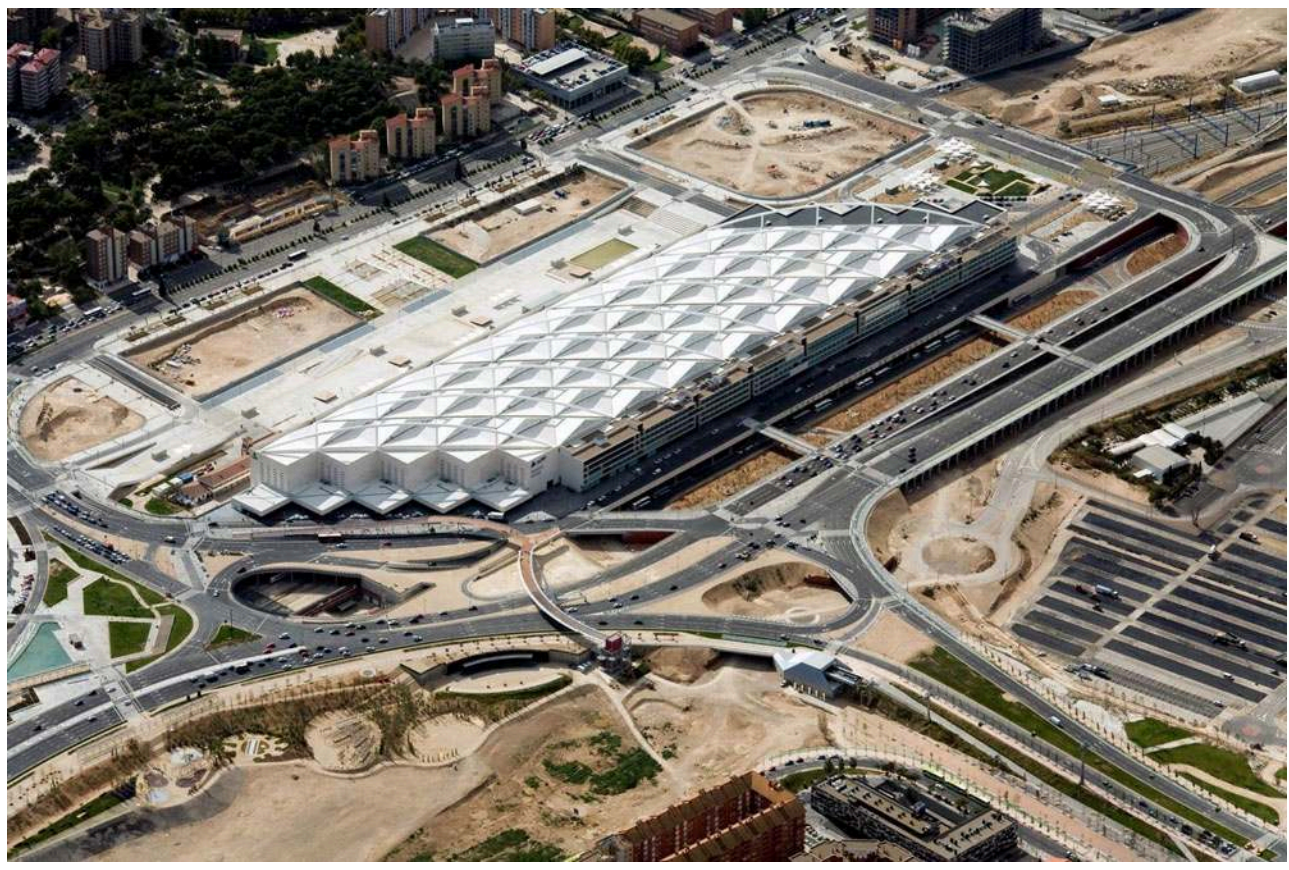

Source : Zaragoza Alta Velocidad

L'urbanisation des abords de la gare de Delicias a nécessité des aménagements d'accessibilité importants comme la fermeture du troisième anneau ferroviaire, la construction d'un nouveau pont sur l'Èbre ou l'aménagement de services ferroviaires de banlieue grâce à l'utilisation d'une partie des infrastructures déjà existantes. Les travaux d'urbanisation et d'accessibilité générale ont beaucoup avancé entre 2003 et 2008, l'année où Saragosse a accueilli l'Exposition internationale qui portait sur le thème de l'eau. L'Exposition a eu lieu dans le méandre de Ranillas, de l'autre côté du fleuve, juste devant la gare de Delicias. C'est pour cette occasion que les travaux autour de la gare avaient progressé, conformément aux plans d'aménagement. Ils consistaient à construire un pont sur l'Ebre, -le pont du troisième millénaire-, des infrastructures d'accès à la gare ainsi qu'à urbaniser des parcs qui menaient au centre-ville.

44 C'est dans la zone du quartier ouest que le projet prévoyait de construire la plupart des 3.000 logements du secteur, créant ainsi un nouveau quartier derrière la gare. Cependant, les travaux du quartier ouest n'ont toujours pas été achevés, de même que la plupart des interventions prévues dans le plan de développement.

45 Des immeubles aux usages divers sont concentrés autour de la gare: des tours de bureaux et de commerces devant la façade principale de la gare et des équipements urbains. 


\section{Le projet Milla Digital (Mille numérique): la ville de l'information et du savoir} sont aujourd'hui la face la plus visible d'un projet ambitieux: Le projet Milla Digital (Mille numérique). Le projet est centré sur la promotion et le développement des TIC (technologies de l'information et de la communication), pour contribuer à l'évolution économique et sociale de Saragosse par le biais de la création d'un quartier où cohabiteraient des logements, des entreprises et des équipements.

\section{Les effets de l'arrivée de la crise sur les projets} environ un milliard d'euros, dans le but de réinvestir toutes les plus-values urbanistiques générées par les ventes des terrains en ville. Cependant, la gestion de ce patrimoine s'avère complexe.

51 Avant même l'arrivée de la crise, des problèmes dans la gestion du patrimoine foncier ont surgi, ainsi que les premiers désaccords entre les différents partenaires relatifs à l'éventualité d'un retour de la propriété du sol si les parcelles n'étaient pas construites dans le délai d'un an. Ces dernières années, les problèmes patrimoniaux et de gestion $\mathrm{du}$ sol ont été résolus mais c'est désormais la crise économique et financière qui paralyse les travaux. Aujourd'hui, aucune parcelle n'a encore été vendue et, par conséquent, toutes les opérations réalisées jusqu'à présent ont été financées par les partenaires de l'accord. La société a donc décidé de suspendre temporairement toutes les opérations jusqu'à ce que des revenus soient disponibles, c'est-à-dire jusqu'à ce que des parcelles soient vendues.

Outre les problèmes liés à la gestion des friches ferroviaires, et à présent à la crise, la société a été accablée par un volume de charges excessif: les projets urbains (et des coûts de financement) allaient bien au-delà des transformations urbaines nécessaires pour préparer la ville à l'arrivée du TGV. En fait, comme nous l'avons expliqué au long du texte, l'arrivée du TGV se présente à la ville comme un grand projet urbain apte à transformer non seulement la structure physique de la ville mais aussi à accompagner le changement de la base économique et la projection de l'image de la ville à l'extérieur

Revue Géographique de l'Est, vol.55 / n³-4 | 2015 
(marketing urbain). Les acteurs ont-ils eu trop tendance à l'époque de la conception des projets urbains à croire à un effet $\mathrm{TGV}$ conjugué à une conjoncture favorable?

La crise immobilière qui a déclenché par la suite la crise économique et financière généralisée a donc mis fin au rêve d'une transformation urbaine rapide. En dehors des travaux ferroviaires, presque tous achevés, de la construction de la gare et des travaux d'accessibilité et d'urbanisation aux alentours de la gare, peu de réalisations prévues dans les projets mentionnés ont été menées à bien.

\section{Conclusions}

Un projet urbain pour la zone métropolitaine de Saragosse a été rédigé et géré par un large partenariat entre différentes parties prenantes et des administrations locales. Il y a toujours eu une grande cohérence entre les exposés de la planification stratégique, le plan d'aménagement urbain et en général les politiques urbaines appliquées dans les années 1990 et 2000. L'un des instruments clé de ce projet urbain a été l'arrivée du TGV : elle a permis de repositionner la ville entre Madrid et Barcelone, elle a révolutionné la mobilité, mais ce qui est plus important est qu'elle a été un puissant instrument pour la transformation physique et fonctionnelle de la ville, pilier sur lequel reposera une bonne partie du projet urbain à venir. Le contexte économique et le soutien des médias ont également contribué à générer une ambiance d'espoir et d'optimisme collectifs. Il n'y avait pas voix visibles d'opposition ni au modèle urbain ni aux ambitieux projets devant être mis au point au plus tard juste après la mise en scène de l'Expo 2008.

Les problèmes dérivés de la gestion du patrimoine foncier ferroviaire, les lourdes charges associées à l'accord (un trop grand nombre d'activités n'ayant que peu de rapport avec le TGV), puis l'arrivée de la crise, semblent avoir paralysé les projets urbanistiques de Saragosse associés à la grande vitesse.

Aujourd'hui Saragosse est quelque peu découragée par rapport à l'avenir du projet "Saragosse grande vitesse". Cependant, il ne faut pas oublier que ces transformations se sont produites dans un temps très court. Les projets mentionnés se sont déroulés au cours des 15 dernières années. Des années de changements vertigineux qui ont été paralysés après l'Exposition universelle et le déclenchement effectif de la crise dans la ville en 2008. Le projet n'est pas entièrement réalisé et il devra être reconsidéré en tenant compte du contexte actuel.

\section{BIBLIOGRAPHY}

Alonso, P., Bellet, C., 2009, «El tren de alta velocidad y el proyecto urbano. Un nuevo ferrocarril para la Zaragoza del tercer milenio», Scripta Nova. Revista Electrónica de Geografía y Ciencias sociales, Vol.XIII, $\mathrm{n}^{\circ} .281$. 
Arnal, J. C., Asín F., Blanco, J. (coord.), 2009, Atlas de la ciudad. Zaragoza 2009, Saragosse, Mairie de Saragosse.

Bel G., Albalate D., 2012, The Economics and Politics of High Speed Rail: Lessons from Experiences Abroad, Lanham: Rowman and Littlefield Publishers, Lexington Books.

Bellet, C., Alonso, P., Casellas, A., 2010, « Infraestructuras de transporte y territorio. Los efectos estructurantes de la llegada del tren de alta velocidad en España», Boletín de la Asociación de Geógrafos Españoles, nº.52, p. 143-163.

Bellet, C., Alonso, P., Gutiérrez, A., 2012, « High-speed rail in Spanish cities: Urban integration and local strategies for socio-economic development», Urena J.M. (ed.), 2012, Territorial implications of High speed rail. A Spanish perspective, Aldershot, Ashgate, p.163-196

Bellet, C., Gutiérrez, A., 2011, «Ciudad y ferrocarril en la España del siglo XXI. La integración de alta velocidad ferroviaria en el medio urbano», Boletín de la Asociación de Geógrafos Españoles, $\mathrm{n}^{\circ}$. 56, p. 251-279.

Bertolini, L., Spit, T., 1998, Cities on rails. The redevelopment of railway station areas, Londres, Spon Press.

Bruinsma, F., Pels, E., Priemus, H., Rietveld, P., Van Wee, B., 2008, Railway development. Impact on urban dynamics, Amsterdam, Physica-Verlag.

Debrezion, G., Willigers, J., 2008, «The effect of railway stations on office space rent levels: The implication of HSL South in station Amsterdam South Axis», Bruinsma, F., Pels, E., Priemus, H., Rietveld, P., Van Wee, B., 2008, Railway development. Impact on urban dynamics, Amsterdam, Physica-Verlag,, p.265-293.

Ebropolis, 2011, Marco Estratégico Zaragoza 2020, consulté en ligne le 1er mai 2014, URL : www.ebropolis.es.

Facchinetti-Mannone, V. 2013, « Les nouvelles gares TGV périphériques: des instruments au service du développement économique des territoires? », Géotransports, 2013, pp.51-66.

$<$ hal-00980008>

Groupe Ten (Courson, J. / Remond,E. / Jaouen,M.) ,1993, Gares TGV et urbanisme. Paris, Société Nationale des chemins de fer français, Ministère de l'équipement, du logement et des transports, 2 vols.

Ménerault, Ph., 2009, «Gares ferroviaires et projets métropolitain: une ville en mutation», POPSU, Lille métropole: laboratoire du renouveau urbain, Marseille, ed. Parenthèses

Ollivro, J., 2006, Quand la Vitesse change le monde, Editions Apogée

Peters, D., Novy, J., 2012, «Rail Station Mega-Projects: Overlooked Centrepieces in the Complex Puzzle of Urban Restructuring in Europe», Built Environment, $n^{\circ} .38$ (1), p. 5-11.

Prada Llorente, E.I., 2010, «Concentración espacial de la propiedad de la tierra, megaproyectos inmobiliarios y transformación del paisaje: caso Valdeluz», Ciudad y Territorio: Estudios Territoriales, vol. XLII, $\mathrm{n}^{\circ} .163$, p. 49-66.

Ureña, J.M., Menerault, P., Garmendia, M., 2009, «The high-speed rail challenge for big intermediate cities: A national, regional and local perspective», Cities, 2009, ${ }^{\circ}$.26, p. 266-279.

Serrano Sanz, J.M., 2009, Los efectos económicos de la Expo Zaragoza 2008, Zaragoza, Fundación Economía Aragonesa.

Troin, F., 1995, Rail et aménagement du territorire, Aix-en-Provence, Edisud. 
Van den Berg, L., Pol, P., 1998, The European high-speed train and urban development. Experiences in fourteen European urban regions, Aldershot, Ashgate.

\section{NOTES}

1. - Les résultats qui sont ici présentés font partie du projet de recherche financé par le MINECO (Ministère de l'Économie et Compétitivité) (Espagne): Patrons de transformation urbaine et stratégies associées à la grande vitesse ferroviaire en Espagne (URBATAV) - CSO2012-34629.

\section{ABSTRACTS}

The project to introduce high-speed rail in Zaragoza coincided with the drafting of a number of strategic and territorial planning documents. In all of these documents, the high-speed train was seen as a key instrument for the urban planning and functional transformation of the city. In this article, we explain how the plans and projects that were supposed to determine the future of the metropolitan area in the 21st century were developed and the role that the new train was to play in them. The onset of the economic crisis frustrated many of the ambitious projects that were associated with the arrival of high-speed rail in the city.

Le projet d'implantation de la grande vitesse ferroviaire à Saragosse a coïncidé dans le temps avec la rédaction de divers documents de planification stratégique et territoriale. Le train à grande vitesse était présenté dans ces documents comme un instrument clé dans la transformation urbanistique et fonctionnelle de la ville. Cet article présente la gestation des plans et des projets qui dessinaient le futur de la zone métropolitaine pour le XXIe siècle et le rôle que le nouveau train allait jouer dans ce contexte. L'arrivée de la crise économique a remis en cause un bon nombre des ambitieux projets associés à l'implantation de la grande vitesse ferroviaire.

Das Projekt für die Einführung von Hochgeschwindigkeitszügen in Zaragoza fiel zeitlich mit der Abfassung mehrerer die Planung und das Gebiet betreffenden Unterlagen zusammen. In allen wurde der Hochgeschwindigkeitszug als ein wesentliches Instrument innerhalb der städtebaulichen und funktionalen Transformation der Stadt dargestellt. In dem Artikel wird gezeigt, wie die Zeichnungen und Entwürfe ausgearbeitet wurden, die die Zukunft des Stadtgebiets für das 21. Jahrhundert und die Rolle, die der neue Zug in allem spielen sollte, sichtbar machten. Der Beginn der Wirtschaftskrise machte einen großen Teil der ehrgeizigen Projekte zunichte, die mit der Einführung der Hochgeschwindigkeitsbahn in der Stadt einhergingen.

\section{INDEX}

Schlüsselwörter. Hochgeschwindigkeitsbahn, städtebaulicher Entwurf, transformation der Stadt Mots-clés: grande vitesse ferroviaire, projet urbain, transformation urbaine Keywords: high-speed rail, urban project, urban transformation 
AUTHOR

CARMEN BELLET SANFELIU

Maître de conférences - Dep. de Géographie et Sociologie, Université de Lleida -

c.bellet@geosoc.udl.cat 